\title{
Cardiorespiratory Phase Synchronization increases during certain mental stimuli in healthy subjects
}

\author{
Jordi Solà-Soler, Member, IEEE; Alba Cuadros and Beatriz F. Giraldo, Senior Member, IEEE
}

\begin{abstract}
Several neurological and mechanical non-linear mechanisms relate the respiratory and cardiovascular systems to one another. Besides the well-known modulation of heart rate by respiration, another form of non-linear interaction between both systems is Cardiorespiratory Phase Synchronization (CRPS). In this study we investigated CRPS on a group of 27 healthy individuals subject to a stimulation protocol with five different mental states: a basal state, a videogame, a comedy video, a suspense video and a reading state. A continuous measure of CRPS was calculated from the phase synchrogram between respiratory and electrocardiographic signals. Periods of CRPS were characterized by their average duration (AvDurSync) and by the percentage of synchronized time (\%Sync) within each mental state. These measures were studied considering two thresholds: a minimum amplitude and a minimum duration for synchronization. Each subject exhibited a particular pattern of phase locking ratios along the different mental states. We observed that, in all states, \%Sync decreased and AvDurSync increased in proportion to the minimum duration threshold. Both measures were inversely proportional to the minimum amplitude threshold. During the videogame, subjects showed a significantly higher \%Sync as compared to any other mental stimulus, irrespective of the minimum duration threshold. Mental stimulation can be an alternative approach to enhance cardiorespiratory coupling when subjects have difficulties to perform aerobic exercise, such as in patients with Chronic Obstructive Pulmonary Disease or Chronic Heart failure.
\end{abstract}

\section{INTRODUCTION}

The cardiovascular and respiratory systems are known to be non-linearly coupled through mechanical and neurological interactions. Cyclical changes in blood pressure and in intrathoracic pressure associated to respiratory motion have a direct and indirect effect on the sino-atrial node. The result is that heart rate increases during inspiration and decreases during exhalation, giving rise to its modulation known as Respiratory Sinus Arrhythmia (RSA) [1]. Simultaneously, cardiac and respiratory rhythms are regulated by the central

This work was supported in part by the Secretariat of Universities and Research of the Department of Economy and Knowledge of the Government of Catalonia (Consolidated research group GRC 2014 SGR 1569), by CERCA Programme / Generalitat de Catalunya, and by the Spanish Ministry of Economy and Competitiveness through project DPI2015-68820-R (MINECO/FEDER).

Alba Cuadros is a Graduated Biomedical Engineer from Escola d'Enginyeria de Barcelona Est (EEBE). J.Solà-Soler and B.F. Giraldo are with Automatic Control Dept. (ESAII), EEBE, Universitat Politècnica de Catalunya (UPC), CIBER de Bioengenieria, Biomateriales y Nanomedicina (CIBER-BBN) and Institute for Bioengineering of Catalonia (IBEC), The Barcelona Institute of Science and Technology, Av. Eduard Maristany, 16. 08019, Barcelona, Spain. (jordi.sola@upc.edu; beatriz.giraldo@upc.edu). nervous system to maintain the homeostatic equilibrium of the body under changing physiological conditions. The neural relation between cardiovascular and respiratory systems is complex and far from being fully understood. But in certain circumstances, these two weakly-coupled self-sustained oscillators may become phase-locked, a type of non-linear coupling known as Cardiorespiratory Phase Synchronization (CRPS). In healthy subjects, periods with high RSA can show either weak or strong CRPS [2]. Therefore, RSA and CRPS reflect two different forms of cardiorespiratory interaction.

In resting conditions, long periods of CRPS were early observed in young, high-performance swimmers [3]. Bartsch and coworkers studied CRPS in healthy subjects during different sleep stages, and they noticed that synchronization is "enhanced by a factor of 2.4 during non-REM sleep", whereas it is "suppressed by a factor of 3 during REM sleep, as compared to wakefulness" [4]. A later study also in healthy subjects reported a higher number of synchronous epochs and longer synchronization periods during a state of inwardattention meditation as compared to a state of normal rest [5]. Altogether, these observations seem to suggest that CRPS is higher during periods of relaxed mental activity.

Previous studies of CRPS during activities involving higher brain regions have obtained different results. Cardiorespiratory phase synchronization was greater during poetry recitation, as compared to periods of controlled breathing exercises or normal rest [6]. On the other hand, performance of mental arithmetic tasks was shown to decrease the duration of cardiorespiratory synchronization epochs [7].

In this work, we study a continuous measure of CRPS in a group of young, healthy individuals. We compare the situation when they are in a resting state, and when they are undergoing different mental tasks.

\section{MATERIAL AND METHODS}

\section{A. Signal Recordings \& Subject database}

The cardiac and respiratory activity was simultaneously recorded in 34 healthy subjects without known pathologies. All subjects gave their written consent to participate in the study. BIOPAC Pro system was used to acquire $30 \mathrm{~min}$ of single lead ECG and thoracic plethysmography band signals at a sampling frequency of $500 \mathrm{~Hz}$ and $100 \mathrm{~Hz}$, respectively. All subjects were studied according to the same stimulation protocol, in which they successively underwent five different stimuli: a basal state (BS), a videogame (GM) -low level for some subjects and high level for the others, a comedy video 
(CV), a suspense video (SV), and finally a reading state (RD) (Figure 1):

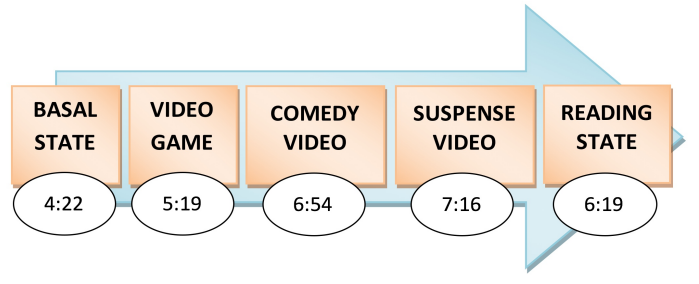

Figure 1. Successive stages in the stimulation protocol and time durations.

Seven subjects had to be excluded because of artifacts in one or both signals. The characteristics of the final database are shown in Table I.

\begin{tabular}{cccc}
\hline \hline \multicolumn{4}{c}{ TABLE I } \\
\hline \hline CHARACTERISTICS OF THE DATABASE \\
\hline PARTICIPANTS & AGE (years) & SEX & $\begin{array}{c}\text { VIDEOGAME } \\
\text { DIFFICULTY }\end{array}$ \\
\hline 27 Subjects & $23.63 \pm 2.75$ & $\begin{array}{c}\text { 21 Male } \\
\text { 6 Female }\end{array}$ & $\begin{array}{c}\text { 16 Easy } \\
\text { 11 Difficult }\end{array}$ \\
\hline \hline
\end{tabular}

\section{B. Signal pre-processing}

The ECG signal was band pass filtered in the range (0.5-35) Hz. The QRS complex was detected using the algorithms provided for free by BioSig library [8]. The extracted R-peak instants $\left\{t_{k}\right\}$ were manually checked and modified whenever necessary. The respiratory signal $r(t)$ was band pass filtered in the range $(0.05-1) \mathrm{Hz}$ with an $8^{\text {th }}$ order Butterworth digital bandpass filter. The respiratory phase $\phi_{r}(t)$ was obtained as

$$
\phi_{r}(t)=\arg [r(t)+j H\{r(t)\}],
$$

where $H\{r(t)\}$ is the Hilbert transform of the respiratory signal defined as

$$
H\{r(t)\}=\frac{1}{\pi} p \cdot v \cdot \int_{-\infty}^{\infty} \frac{r(\tau)}{t-\tau} d \tau .
$$

and $p . v$. stands by Cauchy principal value. The phase signal $\phi_{r}(t)$ was interpolated to obtain the phase value at the $\left\{t_{k}\right\}$ instants, $\phi_{r}\left(t_{k}\right)$.

\section{Phase synchronization estimation}

The synchronization between two weakly coupled oscillators is defined as a locking or entrainment of their phases $\phi_{1}(t)$ and $\phi_{2}(t)$ at some integer ratio $n: m$. In physiological signals, this can be expressed as:

$$
\left|n \phi_{1}(t)-m \phi_{2}(t)\right|<\delta
$$

where $\delta$ is a small value [2]. We implemented a method to obtain a continuous measure of the degree of synchronization between the cardiac and respiratory signals and the periods where it appears [5], [9].

The normalized phase $\psi_{m}\left(t_{k}\right)$ relative to the onset of each respiratory cycle was calculated and plotted against time to obtain the synchrogram:

$$
\psi_{m}\left(t_{k}\right)=\frac{1}{2 \pi}\left[\phi_{r}\left(t_{k}\right) \bmod (2 \pi m)\right],
$$

where $m$ are the respiratory cycles included in each period. The presence of $n$ almost horizontal lines in the representation indicates CRPS of ratio $n: m$, which means that several consecutive groups of $m$ respiratory cycles have the same number of heartbeats $n$. These $n$ horizontal lines can be transformed into a single one by:

$$
\Psi_{n, m}\left(t_{k}\right)=\frac{2 \pi}{m}\left\{\left[\psi\left(t_{k}\right) \cdot n\right] \bmod m\right\} .
$$

The degree of synchronization $\gamma_{n, m}\left(t_{k}\right)$ was calculated by evaluating the invariance of $\Psi_{n, m}\left(t_{k}\right)$ over a defined window:

$$
\gamma_{n, m}=\left\{\frac{1}{N} \sum_{k} \cos \left[\Psi_{n, m}\left(t_{k}\right)\right]\right\}^{2}+\left\{\frac{1}{N} \sum_{k} \sin \left[\Psi_{n, m}\left(t_{k}\right)\right]\right\}^{2}
$$

where $N$ represent the number of heartbeats that happen in the length of the window. The obtained values range between zero (no synchronization) and one (maximum synchronization).

Finally, the maximum degree of synchronization at each instant of time was calculated as

$$
\gamma_{\max }\left(t_{k}\right)=\max _{n, m}\left\{\gamma_{n, m}\left(t_{k}\right)\right\}
$$

Figure 2 shows an example of the synchrogram $\psi_{m}\left(t_{k}\right)$ of a subject and $\gamma_{\max }\left(t_{k}\right)$ obtained during the whole recording:

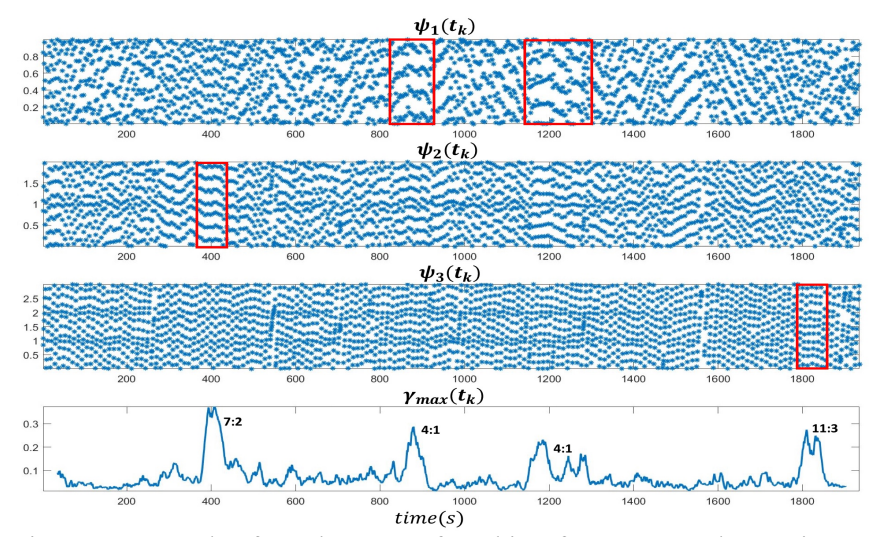

Figure 2. Example of synchrogram of a subject for $m=1,2$ and 3 respiratory cycles. Periods of CRPS of ratios 7:2, 4:1 and 11:3 are all contributing to the final maximum synchronization function.

\section{CRPS characterization}

Synchronization periods were defined as the periods where the degree of synchronization $\gamma_{\max }$ was above a certain threshold. In this study, we compared the threshold values 0.1 and 0.05 . We also studied the effect of establishing a minimum length for a synchronization periods (over the threshold). Previous studies used a minimum synchronization duration of 10s [5]. We compared the performance of the method for minimum lengths of $0 \mathrm{~s}, 5 \mathrm{~s}$ and $10 \mathrm{~s}$.

The CRPS was characterized by the average duration of synchronization (AvDurSync) defined as the arithmetic mean of all synchronized periods, and the percentage of synchronization (\%Sync) as the sum of all periods of synchronization over the duration of the segment. These parameters were calculated for the segment of each stimuli. 


\section{E. Statistical analysis}

The comparison between the different stimuli states were made with Wilcoxon signed-rank test for matched samples, and between the two sexes and the low and high levels of the videogame with Mann-Whitney U test.

\section{RESULTS}

The degree of synchronization at each phase locking ratio $n: m$ and the maximum synchronization over time were represented for all subjects as shown in Figure 3. In general, each subject followed a different pattern. The stimuli segments where synchronization takes place or their exact time locations were not always the same, neither was the distribution of phase locking ratios $n: m$.

In the average \%Sync decreased when the minimum duration period increased (Figure $4 a, b$ ). On the other hand, AvDurSync showed an increasing trend because the periods detected were lower in number but larger in duration (Figure $4 \mathrm{c}, \mathrm{d})$. The two CRPS characteristics were much higher with a
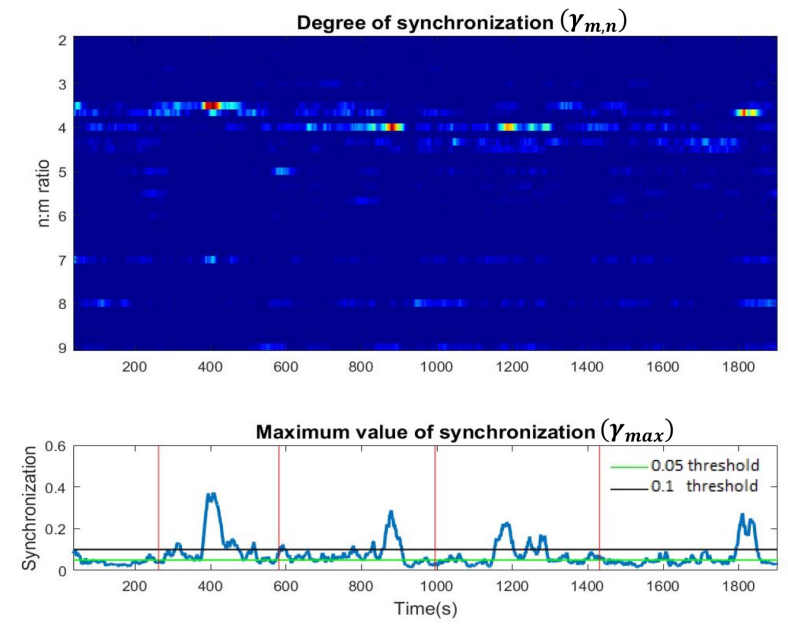

(a) lower synchronization threshold, because there were much more periods over the threshold (Figure 3).

We can observe that the stimulus with the highest \%Sync and AvDurSync was the videogame activity. This happened for all thresholds and minimum duration values (Figures 3, 4).

Statistical comparisons between stimuli for threshold 0.1 showed a significantly higher $\%$ Sync $(\mathrm{p}<0.05)$ during the videogame as compared to any other state, independently of the minimum duration established (Table II b).

AvDurSync was higher during the GM state too, as compared to BS, CV or SV states, but these differences reached statistical significance depending on the minimum duration established (Table II d).

Observing the results for threshold 0.05 (Table II a, c), the videogame and the suspense stages had quite similar \%Sync so these presented statistically significant differences with the other stages but no between them. The statistical results for AvDurSyn at 0.05 also had a different pattern depending on the minimum duration, as it happened for threshold 0.1.
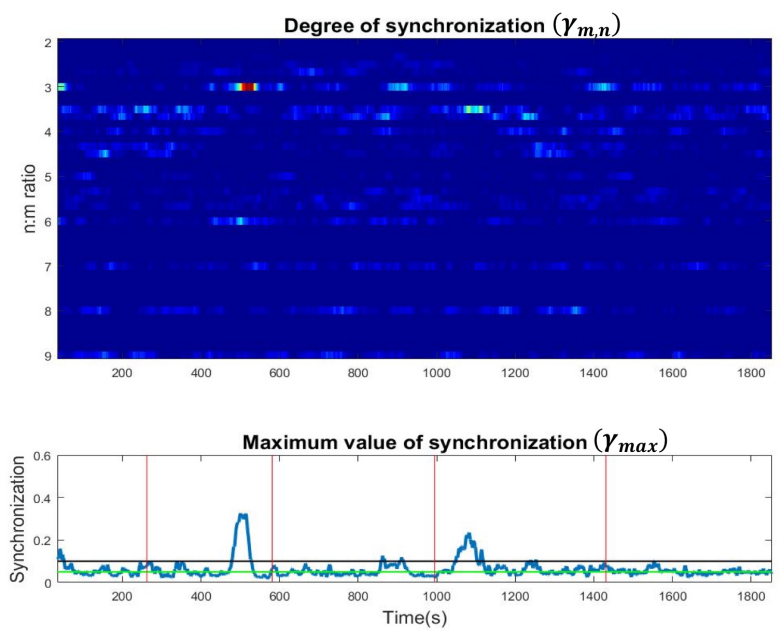

Figure 3. Examples of CRPS evolution over time for two subjects (a) and (b) of the study. Upper panel: Degree of synchronization $\gamma_{n, m}$ for every phase locking ratio $n: m$ during the whole recording time. Lower panel: Maximum value of synchronization calculated at each time instant. Each subject shows synchronization at one or several different phase locking ratios $n: m$. The highest synchronization is observed during the videogame in both subjects.

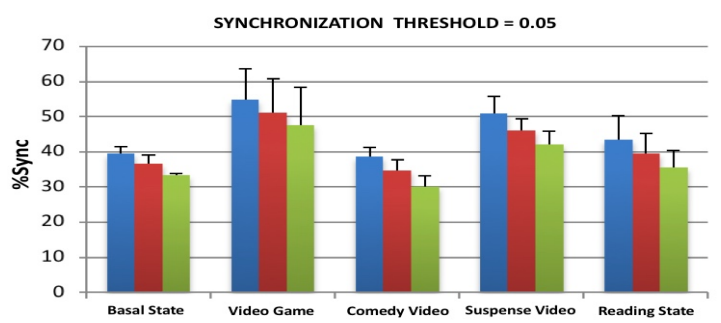

(a)

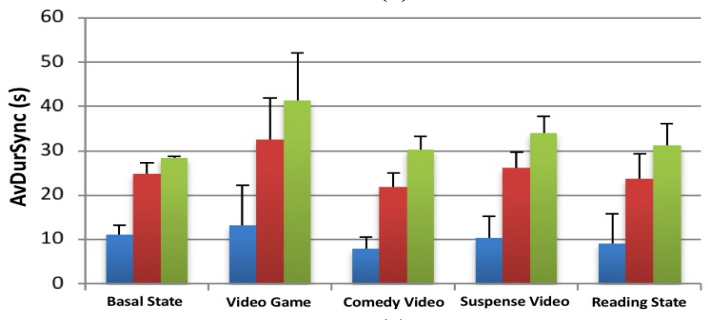

(c)

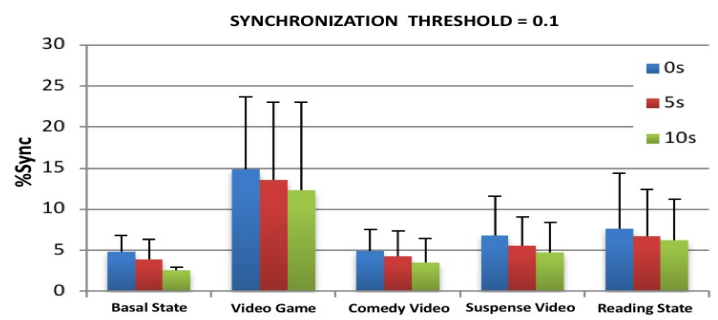

(b)

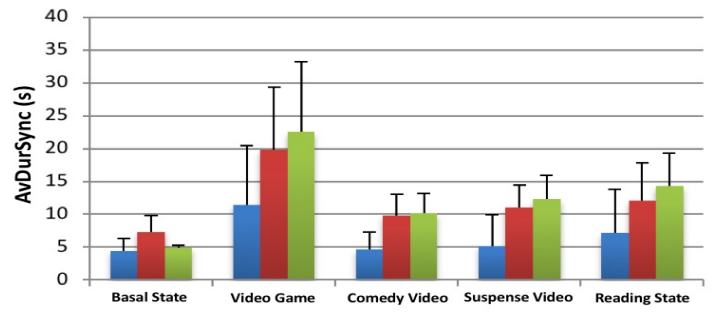

(d)

Figure 4. Percentage of synchronization (\%Sync) and average duration of synchronized periods (AvDurSync) for each stimulus. Increasing the minimum duration from 0 s to 10 s makes the AvDurSync increase and the overall \%Sync decrease. For threshold 0.1 , the basal state shows the lowest values of AvDurSyn and \%Sync, whereas the highest synchronization values occur while playing the videogame. 


\section{SYNCHRONIZATION THRESHOLD $=0.05$}
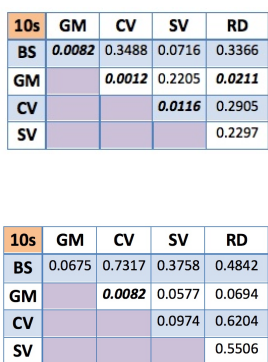

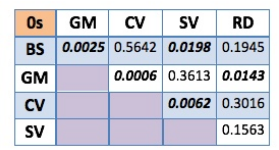

(a) \%Sync (0.05)

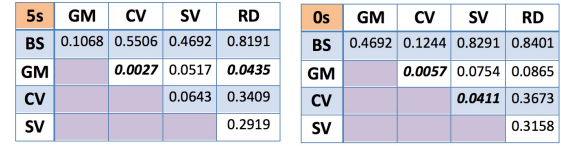

(c) AvDurSync (0.05)

\section{SYNCHRONIZATION THRESHOLD $=0.1$}
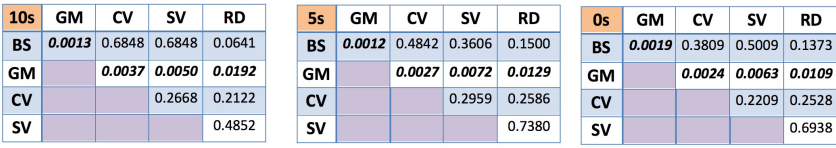

(b) \%Sync (0.1)
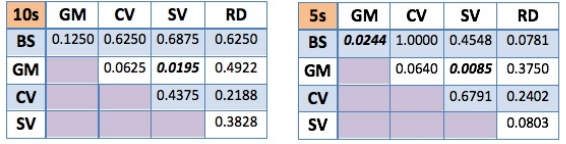

(d) AvDurSync (0.1)

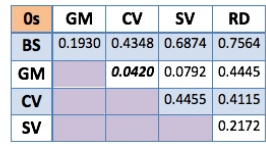

0.2172

TABLE II. Results of the statistical comparison between the different stimuli states: Basal State (BS), Video Game (GM), Comedy Video (CV), Suspense Video (SV) and Reading State (RD). For threshold 0.1 , there are statistically significant differences between GM and all the remaining states, irrespective of the minimum duration of synchronization. These differences are also observed for threshold 0.05, except for the GM-SV comparison.

There were no statistically significant differences in CRPS parameters between men and women for any stimuli. The easy and difficult levels of the videogame did not show any significant difference in CRPS either.

\section{DisCUSSION AND CONCLUSIONS}

Cardiac and respiratory rhythms are directly governed by the autonomous nervous system, but also with influences from the central nervous system. There is a general agreement in that cardiorespiratory coupling, be either in the form of RSA or as CRPS, is a means for optimizing the energy expenditure of the body [1], [2].

The influence of mental state on the percentage and duration of CRPS periods has been attributed mainly to the effect of two factors: long-term correlated noise from higher brain regions, and sympathetic/parasympathetic autonomic balance. Authors who have investigated CRPS as a function of sleep stages propose that, under a relaxed mental state as the one observed in deep sleep, higher brain regions only produce a sort of uncorrelated noise that does not significantly alter autonomous cardiorespiratory interactions. On the other hand, active higher brain states -such as REM sleep- produce a longterm correlated activity that disturbs CRPS [4]. Zhang and colleagues have reported a reduction of cardiorespiratory synchronization while conducting arithmetic mental tasks, which activate the motor cortex. They have analyzed the relationship between heart rate variability and the duration of synchronization periods, and they have found that the decrease in CRPS is significantly correlated with an increase of sympathetic nervous activity [7]. In our experiments it seems that, during the Videogame, participants reach a moment in which the game does not require a high amount of mental effort to them, presumably producing neither mental stress (sympathetic activity) nor correlated noise, therefore allowing CRPS to occur. In fact, Cysarz et al. [6] already reported a significant increase in CRPS during an activity of controlled breathing accompanied by walking through the room and cyclically raising and lowering the arms, as compared to a basal state of rest. This is another kind of activity which involves coordination through higher brain regions while also exhibiting cardiorespiratory synchronization.
Mental stimulation can be an alternative approach to enhance cardiorespiratory coupling in subjects with difficulties to perform aerobic exercise, such as in patients with Chronic Obstructive Pulmonary Disease or Chronic Heart failure. In order to further determine how brain activity influences CRPS, studies of well-defined, randomized mental activities, with simultaneous recording of cardiorespiratory signals and brain electrical activity, could be very helpful.

\section{ACKNOWLEDGMENT}

The authors would like to thank to B. Martínez and S. Antolino for developing the protocol and recording the signals for this work, and to all volunteers who participated in these recordings.

\section{REFERENCES}

[1] F. Yasuma and J. I. Hayano, "Respiratory Sinus Arrhythmia: Why Does the Heartbeat Synchronize with Respiratory Rhythm?," Chest, vol. 125, no. 2, pp. 683-690, 2004.

[2] R. P. Bartsch, A. Y. Schumann, J. W. Kantelhardt, T. Penzel, and P. C. Ivanov, "Phase transitions in physiologic coupling," Proc. Natl. Acad. Sci., vol. 109, no. 26, pp. 10181-10186, 2012.

[3] C. Schäfer, M. G. Rosenblum, H. H. Abel, and J. Kurths, "Synchronization in the human cardiorespiratory system.," Phys. Rev. E. Stat. Phys. Plasmas. Fluids. Relat. Interdiscip. Topics, vol. 60 , no. 1, pp. 857-870, 1999 .

[4] R. Bartsch, J. W. Kantelhardt, T. Penzel, and S. Havlin, "Experimental evidence for phase synchronization transitions in the human cardiorespiratory system," Phys. Rev. Lett., vol. 98(5), no. 5, 2007

[5] S. Da Wu and P. C. Lo, "Cardiorespiratory phase synchronization during normal rest and inward-attention meditation," Int. J. Cardiol., vol. 141, no. 3, pp. 325-328, 2010.

[6] D. Cysarz, D. von Bonin, H. Lackner, P. Heusser, M. Moser, and H. Bettermann, "Oscillations of heart rate and respiration synchronize during poetry recitation.," Am. J. Physiol. Heart Circ. Physiol., vol. 287, no. 2, pp. H579-87, 2004.

[7] J. Zhang, X. Yu, and D. Xie, "Effects of mental tasks on the cardiorespiratory synchronization," Respir. Physiol. Neurobiol., vol. 170, no. 1, pp. 91-95, 2010.

[8] A. Schlögl and C. Brunner, "BioSig: A free and open source software library for BCI research," Computer (Long. Beach. Calif)., vol. 41, no. 10, pp. 44-50, 2008.

[9] A. Kuhnhold et al., "Quantifying cardio-respiratory phase synchronization - A comparison of five methods using ECGs of post-infarction patients," Physiol. Meas., vol. 38, no. 5, pp. 925939, 2017. 